\title{
Menstrual Cycle does not Affect the Mechanical Properties of Muscle and Tendon
}

\author{
S. Taş ${ }^{1}, \mathrm{D}$. Aktaş ${ }^{2}$ \\ 1 Toros University, School of Health Sciences, Department of Physiotherapy and Rehabilitation, Mersin, Turkey \\ 2 Çankırı Karatekin University, Faculty of Health Science, Department of Midwifery, Çankırı, Turkey
}

\section{CORRESPONDING AUTHOR:}

\section{Serkan Taş}

Toros University, School of Health Sciences

Department of Physiotherapy and

Rehabilitation

33140, Mersin, Turkey

E-mail: serkntas@gmail.com

Phone: +903243253300

Fax: +90 3243253301

DOI:

10.32098/mltj.01.2020.02

LEVEL OF EVIDENCE: 3B

\begin{abstract}
SUMMARY
Background. The evidences suggest that the risk of orthopaedic injury is the highest in the ovulation phase of the menstrual cycle. The change on mechanical properties of muscle and/or tendon related the higher estrogen concentration in the ovulation phase may be related to the highest risk of orthopaedic injury in ovulation phase. The purpose of the study was to investigate the menstrual cycle effect on the stiffness, elasticity and/or tone of the medial gastrocnemius muscle, Achilles and patellar tendons.

Methods. The study was carried out in 30 healthy sedentary young females. The stiffness, elasticity and/or tone of the medial gastrocnemius muscle, Achilles and patellar tendons were measured using a MyotonPRO device. The stiffness, elasticity and/or tone of selected muscle and tendons were measured in the follicular, ovulatory, and luteal phases to determine the menstrual cycle variation.

Results. There was no significant difference in stiffness, elasticity and tone of the Achilles tendon, patellar tendon and medial gastrocnemius muscle among phases $(p>.05)$. Conclusions. The results suggest that the menstrual cycle do not affect the stiffness, elasticity and/or tone of the medial gastrocnemius muscle, Achilles and patellar tendons.
\end{abstract}

\section{KEY WORDS}

Elasticity; menstrual cycle; muscle; stiffness; tendon; tone

\section{BACKGROUND}

Females have higher the risk of orthopaedic injuries during athletic performance and recreational activities than males $(1,2)$. In addition, incidence of many degenerative orthopaedic diseases such as osteoarthritis in females is significantly higher compared to males (3). It is through that one of the contributing factors for higher incidence or risk of orthopaedic injury in females is sex difference in hormone concentrations especially estrogen concentration $(4,5)$. In addition, the evidences suggest that the risk of orthopaedic injury is the highest in ovulation phase of the menstrual cycle, in which is the highest estrogen concentration $(4,5)$. For example, Balachandar et al. (4) revealed that females are at the greatest risk of anterior cruciate ligament injury during pre-ovulatory phase because of greater ACL laxity, greater knee valgus, and greater tibial external rotation during functional activity. However, it is not completely known which factors decrease the joint stability in ovulation phase.

The changes in mechanical properties of muscle and/ or tendon related the higher estrogen concentration in ovulation phase may be a factor affecting the joint stability. Estrogen is an important hormone for musculoskeletal tissues homeostasis, and an increase in estrogen concentration may cause a decrease in collagen synthesis $(6,7)$. In theory, decrease in collagen synthesis could lead to change the mechanical properties of muscle, tendon and ligament $(8,9)$, and it may cause a decrease in neuromuscular control and/or joint stability (10). Consequently, it may lead to predispose to orthopaedic injury. Indeed, there are some attempt which investigated the effect of menstrual cycle on mechanical properties of muscle and/or tendon, but the results of these studies are contradicting. Some of these studies revealed a decrease in muscle stiffness and/ 
or elasticity in ovulation phase $(11,12)$; however, the other reported no significant difference in muscle stiffness and/ or elasticity among the menstrual phases $(13,14)$. Furthermore, there are limited study about menstrual cycle effect on tendon mechanical properties, and they used indirect methods to determine the differences in tendon mechanical properties $(15,16)$. Identifying the possible differences on mechanical properties of muscle and tendon during menstrual cycle may help us to better understand the higher risk of orthopaedic injuries in females and also to develop better preventive or treatment strategies. Therefore, the purpose of the present study was to investigate the differences in the elasticity, stiffness and/or tone of medial gastrocnemius muscle, Achilles tendon and patellar tendon among the menstrual cycle. We hypothesized that the elasticity, stiffness and/or tone of medial gastrocnemius muscle, Achilles tendon and patellar tendon would be lower in ovulation phase compared follicular, and luteal phases.

\section{MATERIAL AND METHODS}

\section{Sample size calculation}

The sample size calculation was performed using the SPSS Sample Power 3.0 software (IBM Corporation, Armonk, NY). It was estimated that at least 21 females had to be included in this study for $80 \%$ power with $5 \%$ type- 1 error level to detect a minimum significant difference of $35 \mathrm{~N} \cdot \mathrm{m}$ for muscle stiffness among menstrual phases, when the average value of muscle stiffness in follicular phase is $242 \mathrm{~N} \cdot \mathrm{m}$, with a standard deviation of $35 \mathrm{~N} \cdot \mathrm{m}(12)$.

\section{Participants}

The study was carried out in 30 healthy sedentary young females with a normal weigh [age 21.3 (1.6) years; height $1.66(0.05) \mathrm{m}$; weight $55.4(6.0 \mathrm{~kg})$; body mass index 20.2 (1.9) $\left.\mathrm{kg} / \mathrm{m}^{2}\right]$. Females, whose body mass index were under $25 \mathrm{~kg} / \mathrm{m}^{2}$, were accepted as a normal weight. Overweight and obese females were excluded the study, because high level in superficial adipose tissue may cause a decrease in reliability and validity of the mechanical property measurements. Females, were had not performed regularly exercise for at least 6 months prior to the present study, excepted as sedentary individuals. Females were excluded from the study if they met any of the following exclusion criteria: (1) had a history of pregnancy; (2) had a history of contraceptive pill use; (3) had an abnormal duration of menses $(<4$ day, and $>7$ days $)$ and/or menstrual cycle $(<$
26 day, and $>30$ days); (4) had a history of lower extremity injuries, major trauma and/or surgery; (5) had a rheumatic and/or systemic disease. The present study was approved by the ethics committee of our institution. All participants provided oral and written informed consent prior to experiment.

\section{Experimental protocol}

Similar to previous studies $(14,17)$, the menstrual cycle was classified into 3 phases; follicular, ovulatory, and luteal phases (1-9 days, 10-14 days, and 15-28 days from the first day of menses, respectively). Mechanical properties of selected muscle and tendons were measured in the central days of the 3 phases. The measurements were performed at the days between 4 and 6 days in follicular phase, 10-14 days in ovulatory phase, and 21-25 days in luteal phase considering the first day of menses. Researcher, who performed the mechanical properties measurements of the selected tissues, were blinded to the menstrual cycle of the subjects.

\section{Mechanical properties measurements}

The mechanical properties measurements of medial gastrocnemius muscle, Achilles and patellar tendons were performed using a MyotonPRO device. It was reported that MyotonPRO device is reliable and valid for measuring the mechanical properties of muscle and tendon (18-20). The device measures mechanical oscillations of assessed soft tissues by a mechanical impulse which is short duration (15 milliseconds) and involves a constant mechanical force (up to $0.6 \mathrm{~N}$ ). Measuring the mechanical oscillations occurring due to mechanical impulse yields the following data: oscillation frequency $(\mathrm{Hz})$, logarithmic decrement and stiffness $(\mathrm{N} \cdot \mathrm{m})$. Oscillation frequency $(\mathrm{Hz})$ characterises the tone (intrinsic tension on the cellular level) of a muscle in its passive or resting state without any voluntary contraction. Dynamic stiffness $(\mathrm{N} \cdot \mathrm{m})$ characterises the resistance to an external force or to a contraction. Logarithmic decrement characterises its elasticity. Elasticity characterises the ability to recover its initial shape after a contraction or removal of an external force of deformation.

Mechanical properties of muscle and tendon in the resting state were measured on right leg. The measurements were carried out at the same time of the day $( \pm 1$ h) by the same operator. In order to prevent confounding effects, the participants were asked not to do any strenuous exercises within the $24 \mathrm{~h}$ prior to assessments. Based on previous studies (18-20), mechanical properties of the Achilles tendon and medial gastrocnemius muscle were measured 
when the individuals in prone position with knee extension and neutral ankle position. The elasticity and stiffness measurements of the Achilles tendon were measured at the point by $2 \mathrm{~cm}$ proximal to the superior aspect of the calcaneus, because pathological changes in the Achilles tendon is commonly seen in this area (21). The tone, elasticity and stiffness measurement of the medial gastrocnemius muscle were performed at $30 \%$ of the lower leg length distal in line with medial malleolus to medial joint of the knee. Similar to previous studies (22), the mechanical properties measurements of the patellar tendon were performed when the individuals in supine position with knee flexed at $30^{\circ}$. The stiffness and elasticity of the patellar tendon were measured at the proximal part of the tendon (point by $2 \mathrm{~cm}$ distal of the inferior pole of patella) (figure 1 ). The point was selected because pathological changes in patellar tendon is commonly seen in this area (23). Mechanical properties of assessed tissues ware calculated by taking the average of three successive measurements performed.

\section{Statistical analysis}

Statistical analyses were performed using a statistic software (SPSS version 22, IBM, New York, USA). The variables were investigated using visual (histograms and probability plots) and analytical methods (Kormogorov-Smirnov/ Shapiro-Wilk's test) to determine whether or not they are normally distributed. For normally distributed variables, mean and standard values were presented. Repeated measures ANOVA was used to determine the differences in the mechanical properties of tendon and muscle among menstrual phases. Paired-Samples T-test was used to compare the mechanical properties of the gastrocnemius muscle, Achilles and patellar tendon in follicular phase. An overall \%5 Type-I error level was used to infer statistical significance.

\section{RESULTS}

The mechanical properties in follicular, ovulatory, and luteal phases were measured on 4.8 (0.8) days, 12.0 (0.6) and 23.8 (1.3) days from the first day of menses, respectively. It was found that oscillation frequency values of the Achilles tendon, patellar tendon and medial gastrocnemius muscle were similar in the follicular, ovulatory, and luteal phases $(p>.05)$. Similarly, there was no significant difference in stiffness of the Achilles tendon, patellar tendon and medial gastrocnemius muscle among phases ( $p$ > .05). Furthermore, the menstrual cycle had no significant effect in logarithmic decrement values of the Achilles tendon, patellar tendon and medial gastrocnemius muscle (p > .05) (table I).

The paired $\mathrm{T}$ test showed that logarithmic decrement value of the Achilles tendon was lower than the patellar tendon $(\mathrm{p}<.001)$, while the Achilles tendon had higher stiffness value compared to the patellar tendon $(\mathrm{p}<.001)$. Moreover, the medial gastrocnemius muscle stiffness was lower than the Achilles and patellar tendons $(\mathrm{p}<.001)$, while the gastrocnemius muscle elasticity was higher than the Achilles tendon $(\mathrm{p}<.001)$ (figure 2$)$.
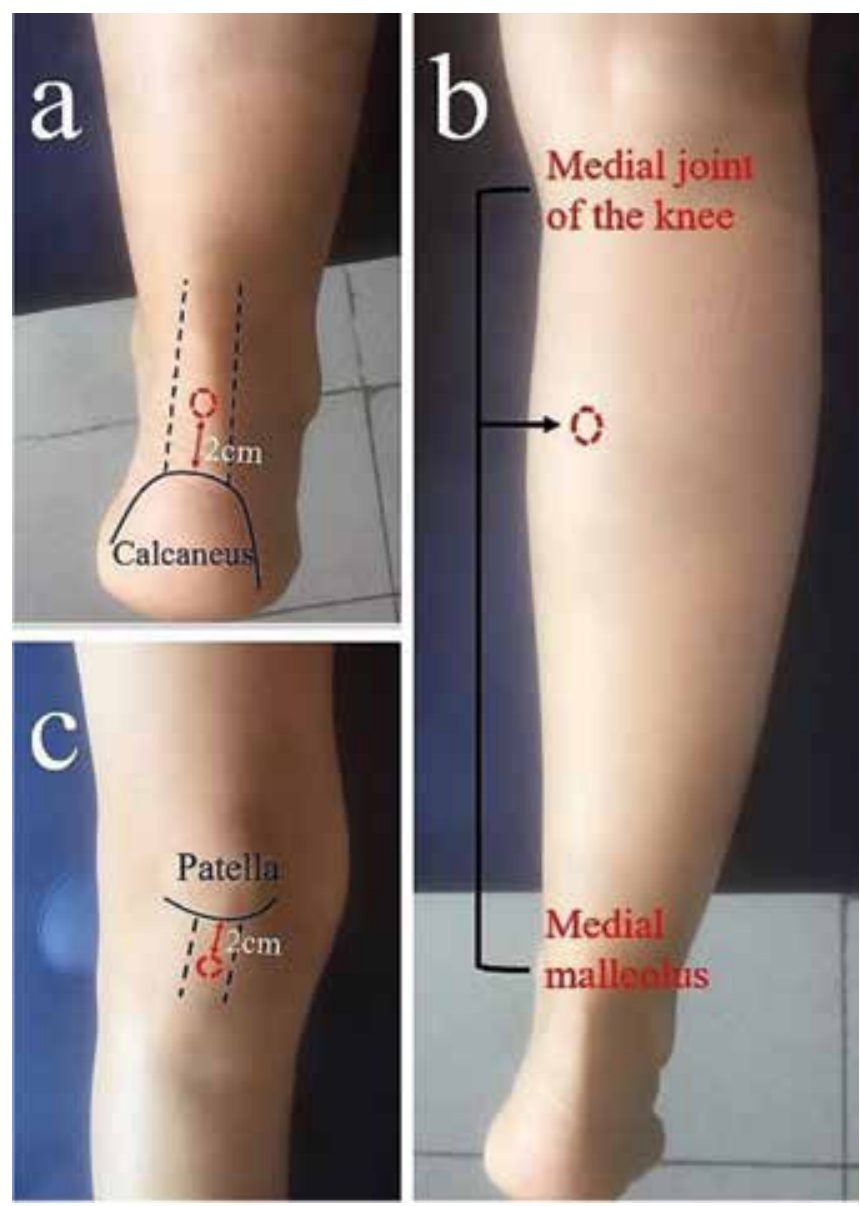

Figure 1. Measurement point on Achilles tendon, patellar tendon and medial gastrocnemius muscle. (a) The elasticity and stiffness measurements of Achilles tendon were measured at the point of $2 \mathrm{~cm}$ proximal to the superior aspect of the calcaneus; (b) The tone, elasticity and stiffness measurement of the medial gastrocnemius muscle were performed at 30 $\%$ of the lower leg length distal in line with medial malleolus to medial joint of the knee; (c) The stiffness and elasticity of patellar tendon were measured at the proximal part of the tendon (point of $2 \mathrm{~cm}$ distal of the inferior pole of patella). 
Table I. Mechanical properties values of Achilles tendon, patellar tendon and medial gastrocnemius in follicular, ovulatory and luteal phases $(n=30)$. Data are presented as mean (standard deviation).

\begin{tabular}{lcccc}
\hline Parameters & Follicular phase & Ovulatory phase & Luteal phase & P \\
\hline Achilles tendon & & & & \\
\hline Oscillation frequency $(\mathrm{Hz})$ & $31.0(2.2)$ & $31.2(1.9)$ & $31.5(1.2)$ & .387 \\
\hline Logarithmic decrement & $0.8(0.1)$ & $0.8(0.1)$ & $0.8(0.1)$ & .293 \\
\hline Stiffness $(\mathrm{N} \cdot \mathrm{m})$ & $790.0(83.0)$ & $805.7(62.9)$ & $817.6(51.1)$ & .141 \\
\hline Patellar tendon & & & \\
\hline Oscillation frequency $(\mathrm{Hz})$ & $17.7(2.0)$ & $17.6(1.9)$ & $17.6(2.0)$ & .921 \\
\hline Logarithmic decrement & $1.0(0.1)$ & $1.0(0.1)$ & $1.0(0.1)$ & .878 \\
\hline Stiffness $(\mathrm{N} \cdot \mathrm{m})$ & $367.8(79.7)$ & $363.3(79.3)$ & $368.6(80.3)$ & .435 \\
\hline Medial gastrocnemius muscle & & & & .876 \\
\hline Oscillation frequency $(\mathrm{Hz})$ & $14.8(1.8)$ & $14.7(1.4)$ & $14.7(1.5)$ & .260 \\
\hline Logarithmic decrement & $1.1(0.2)$ & $1.0(0.2)$ & $1.0(0.1)$ & .565 \\
\hline Stiffness $(\mathrm{N} \cdot \mathrm{m})$ & $257.0(52.5)$ & $252.5(38.6)$ & $255.9(43.4)$ &
\end{tabular}

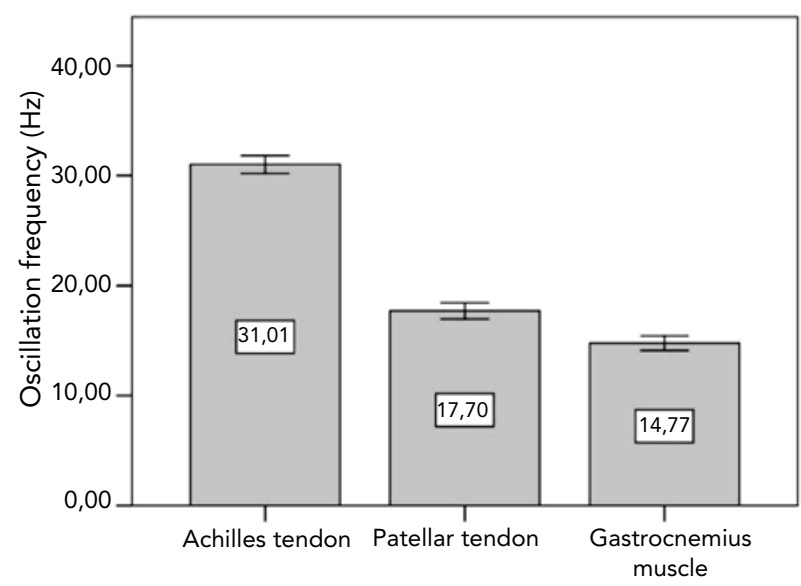

\section{DISCUSSION}

The purpose of the present study was to investigate the effect of menstrual cycle on mechanical properties of muscle and tendon. We hypothesized that stiffness, elasticity and/ or tone of assessed tissues would be lower in the ovulatory phase compared to the follicular and luteal phases. The hypothesis based on the idea that estrogen level is the highest in the ovulatory phase, and high estrogen level could cause collagen loss $(6,7)$, which could affect the mechanical properties of muscle and tendon $(6,8,9)$. Different from our hypothesis, we found that the menstrual cycle had no effect on the stiffness, elasticity and/or tone of the Achilles
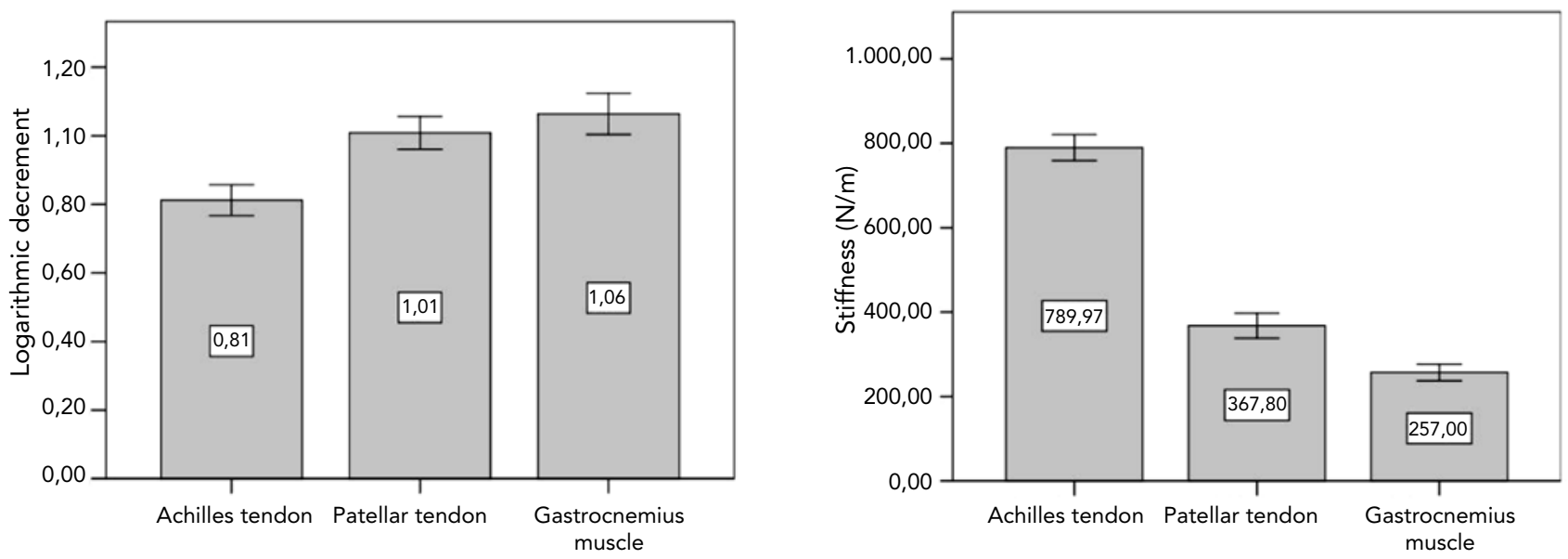

Figure 2. Stiffness, logarithmic decrement and oscillation frequency values of Achilles tendon, patellar tendon and medial gastrocnemius muscle in follicular phase. 
tendon, patellar tendon and medial gastrocnemius muscle. There are some studies which investigated the menstrual cycle effect on tissue stiffness, but they predominantly focused on the muscle stiffness. Saeki et al. (14) investigated the menstrual cycle effect on the triceps surae muscle by shear-wave elastography. Similar to our results, they reported that menstrual cycle did not affect the stiffness of the triceps surae muscle. On the other hand, Yim et al. (11) quantified the muscle tone, elasticity and stiffness of lateral gastrocnemius, tibialis anterior and peroneus longus muscles in the follicular and ovulation phases by a portable myotonometer. They reported that tone of assessed muscles was significantly higher in follicular phase than ovulation phase; however, muscle stiffness was similar in both menstrual phases. Bell et al. (13) examined the hamstring stiffness and extensibility in menstrual phases by modelling the knee as a single degree-of-freedom mass spring system and measuring maximal passive hip flexion. They revealed that hamstring stiffness did not change across the menstrual cycle; however, hamstring extensibility increased in ovulation phase. Moreover, Sung and Kim (12) reported that muscle tone and stiffness of the vastus medialis and semitendinosus muscles were higher in ovulation phase compared to follicular and luteal phases; however, menstrual cycle did not affect the muscle tone and stiffness of the vastus lateralis and biceps femoris muscles. Lee at al. (24) investigated the changes in anterior cruciate ligament elasticity and estradiol concentration among the menstrual cycles. They reported an increase in estradiol concentration and anterior cruciate ligament elasticity during ovulation phase. Balachandar et al. 4 revealed that females are at the greatest risk of anterior cruciate ligament injury during pre-ovulatory phase because of greater ACL laxity, greater knee valgus, and greater tibial external rotation during functional activity. On the other hand, Stefani et al. (25) reported that menstrual disorders did not affect the body composition and cardiac functional pattern in athletes. It seen that there are conflicted results about menstrual cycle effect on muscle stiffness.

It might be expected that menstrual cycle effect must be more significant in tendon mechanical properties compared to muscle considering estrogen effect in collagen concentration, because collagen concentration of tendon contains is fairly higher than muscle. However, obtained results suggest that menstrual cycle does not have any effect on stiffness and elasticity of the Achilles and patellar tendons. There is limited study which investigated the effect of menstrual cycle on tendon mechanical properties. However, tendon stiffness was measured with indirect methods in previous studies, and other mechanical properties such as elasticity and/or tone was not investigated. In a study, Kubo et al. (15) investigated the change in tendon stiffness on menstru- al phases with calculating elongations of the tendon structures in knee extensors and plantar flexors during isometric contraction by ultrasonography. They found that tendon stiffness was similar in follicular, ovulatory, and luteal phases. Burgess et al. (16) reported that there was no significant difference among menstrual cycle in the mechanical properties of medial gastrocnemius tendon calculated elongations of the gastrocnemius fascicle and the aponeurosis during isometric contraction. Furthermore, Pearson et al. (26) indicated that mechanical properties of the patellar and gastrocnemius tendons were similar in follicular, ovulatory, and luteal phases. Different from menstrual cycle effect on muscle mechanical properties, there is a consensus about menstrual cycle effect on tendon mechanical properties.

The obtained results revealed that the Achilles tendon stiffness was two times more than the patellar tendon stiffness; however, the Achilles tendon elasticity was lower than the patellar tendon elasticity. In addition, the medial gastrocnemius muscle stiffness was lower than the patellar and Achilles tendon. Coombes et al. (27) examined the Achilles and patellar tendon stiffness by shear-wave elastography. Similar our results, they reported that the Achilles tendon stiffness (mean:10.4 $\pm 1.1 \mathrm{~m} / \mathrm{s}$ ) was higher than the patellar tendon stiffness (mean: $6.4 \pm 1.2 \mathrm{~m} / \mathrm{s}$ ). Furthermore, Cristi-Sánchez et al. (28) investigated the Achilles and patellar tendon stiffness in health participant using MyotonPRO. They reported that the Achilles and patellar tendon stiffness in dominant leg were $1098.0 \pm 139.5 \mathrm{~N} \cdot \mathrm{m}$ and $897.7 \pm 190.1 \mathrm{~N}$ - $\mathrm{m}$, respectively. Difference from obtained stiffness values of patellar tendon may be related to selected measurement position. Cristi-Sánchez et al. (28) used $90^{\circ}$ knee flexion for measuring patellar tendon stiffness in males and females; however, we used $30^{\circ}$ knee flexion position in only females. Tension in patellar tendon is higher in $90^{\circ}$ knee flexion, and it could lead to obtain higher stiffness value. On the other hand, standard deviations of obtained mean values were in a wide range; however, the study included healthy, sedentary and young females with normal weight. The results suggest that individual differences have important effect on mechanical properties of muscle and tendon.

The present study has some limitation. First, the study was conducted with healthy and young females. The menstrual cycle effect on the muscle and/or tendon may be different in different population, and pathological condition (29). Second, hormone concentration was not measured to classify the menstrual cycle phase. However, all participants had a normal menstrual cycle. Therefore, we think that this situation did not have a significant effect on the obtained result. It was found that elasticity, stiffness and/or tone of medial gastrocnemius muscle, Achilles tendon and patellar tendon were similar in follicular, ovulatory, and luteal phases. The 
results suggest that menstrual cycle does not affect the mechanical properties of assessed muscle and tendons. In addition, Achilles tendon stiffness was higher; however, Achilles tendon elasticity was lower than patellar tendon.

\section{REFERENCES}

1. Moore E, Chalmers S, Milanese S, Fuller JT. Factors influencing the relationship between the functional movement screen and injury risk in sporting populations: a systematic review and meta-analysis. Sports Med. 2019;49:1449-1463.

2. Ceyssens L, Vanelderen R, Barton C, Malliaras P, Dingenen B. Biomechanical risk factors associated with running-related injuries: a systematic review. Sports Med. 2019;49:1095-1115

3. Vina ER, Kwoh CK. Epidemiology of osteoarthritis: literature update. Curr Opin Rheumatol. Curr Opin Rheumatol 2018;30:160-167.

4. Balachandar V, Marciniak JL, Wall O, Balachandar C. Effects of the menstrual cycle on lower-limb biomechanics, neuromuscular control, and anterior cruciate ligament injury risk: a systematic review. Muscles Ligaments Tendons. J 2017;7:136-146.

5. Eiling E, Bryant AL, Petersen W, Murphy A, Hohmann E. Effects of menstrual-cycle hormone fluctuations on musculotendinous stiffness and knee joint laxity. Knee Surg Sports Traumatol Arthrosc. 2007;15:126-32.

6. Wentorf FA, Sudoh K, Moses C, Arendt EA, Carlson CS. The effects of estrogen on material and mechanical properties of the intra- and extra-articular knee structures. Am J Sports Med. 2006;34:1948-52..

7. Hart DA, Archambault JM, Kydd A, Reno C, Frank CB, Herzog W. Gender and neurogenic variables in tendon biology and repetitive motion disorders. Clin Orthop Relat Res. 1998;351:44-56.

8. Gajdosik RL. Passive extensibility of skeletal muscle: review of the literature with clinical implications. Clin Biomech (Bristol, Avon). 2001;16:87-101.

9. Purslow PP. Strain-induced reorientation of an intramuscular connective tissue network: implications for passive muscle elasticity. J Biomech. 1989;22:21-31.

10. Knutson GA. The role of the gamma-motor system in increasing muscle tone and muscle pain syndromes: a review of the Johansson/Sojka hypothesis. J Manipulative Physiol Ther. 2000;23:564-72.

11. Yim J, Petrofsky J, Lee H. Correlation between mechanical properties of the ankle muscles and postural sway during the menstrual cycle. Tohoku J Exp Med. 2018;244:201-207.

12. Sung ES, Kim JH. The difference effect of estrogen on muscle tone of medial and lateral thigh muscle during ovulation. J Exerc Rehabil. 2018;14:419-423.

13. Bell DR, Myrick MP, Blackburn JT, Shultz SJ, Guskiewicz KM, Padua DA. The effect of menstrual-cycle phase on hamstring extensibility and muscle stiffness. J Sport Rehabil. 2009;18:553-63.

14. Saeki J, Ikezoe T, Yoshimi S, Nakamura M, Ichihashi N. Menstrual cycle variation and gender difference in muscle stiffness of triceps surae. Clin Biomech (Bristol, Avon). 2019;61:222-226.

15. Kubo K, Miyamoto M, Tanaka S, Maki A, Tsunoda N, Kanehisa H. Muscle and tendon properties during menstrual cycle. Int J Sports Med. 2009;30:139-43.

\section{CONFLICT OF INTERESTS}

The authors declare that they have no conflict of interests.

16. Burgess KE, Pearson SJ, Onambele GL. Menstrual cycle variations in oestradiol and progesterone have no impact on in vivo medial gastrocnemius tendon mechanical properties. Clin Biomech (Bristol, Avon). 2009;24:504-9.

17. Karageanes SJ, Blackburn K, Vangelos ZA. The association of the menstrual cycle with the laxity of the anterior cruciate ligament in adolescent female athletes. Clin J Sport Med. 2000;10:162-168.

18. Frohlich-Zwahlen AK, Casartelli NC, Item-Glatthorn JF, Maffiuletti NA. Validity of resting myotonometric assessment of lower extremity muscles in chronic stroke patients with limited hypertonia: a preliminary study. J Electromyogr Kinesiol. 2014;24:762-9.

19. Taş S, Salkın Y. An investigation of the sex-related difference in stiffness of achilles tendon and gastrocnemius muscle: Inter-observer and inter-day reliability and the effect of ankle joint motion. The Foot. 2019:41;44-50.

20. Chen G, Wu J, Chen G, et al. Reliability of a portable device for quantifying tone and stiffness of quadriceps femoris and patellar tendon at different knee flexion angles. PLoS One. 2019;14:e0220521.

21. Peltonen J, Cronin NJ, Avela J, Finni T. In vivo mechanical response of human Achilles tendon to a single bout of hopping exercise. J Exp Biol. 2010;213:1259-65.

22. Tas S, Yilmaz S, Onur MR, Soylu AR, Altuntas O, Korkusuz F. Patellar tendon mechanical properties change with gender, body mass index and quadriceps femoris muscle strength. Acta Orthop Traumatol Turc. 2017;51:54-59.

23. Johnson DP, Wakeley CJ, Watt I. Magnetic resonance imaging of patellar tendonitis. J Bone Joint Surg Br. 1996;78:452-7

24. Lee H, Petrofsky JS, Daher N, Berk L, Laymon M, Khowailed IA. Anterior cruciate ligament elasticity and force for flexion during the menstrual cycle. Med Sci Monit. 2013;19:1080-8.

25. Stefani L, Galanti G, Lorini S, Beni G, Dei M, Maffulli N. Female athletes and menstrual disorders: a pilot study. Muscles Ligaments Tendons J. 2016;6:183-187.

26. Pearson SJ, Burgess KE, Onambélé GL. Serum relaxin levels affect the in vivo properties of some but not all tendons in normally menstruating young women. Exp Physiol. 2011;96(7):681-8.

27. Coombes BK, Tucker K, Vicenzino B, et al. Achilles and patellar tendinopathy display opposite changes in elastic properties: A shear wave elastography study. Scand J Med Sci Sports. 2018;28:1201-1208.

28. Cristi-Sanchez I, Danes-Daetz C, Neira A, Ferrada W, Yanez Diaz R, Silvestre Aguirre R. Patellar and Achilles tendon stiffness in elite soccer players assessed using myotonometric measurements. Sports Health. 2019;11:157-162.

29. Padulo J, Oliva F, Frizziero A, Maffulli N. Muscles, Ligaments and Tendons Journal - Basic principles and recommendations in clinical and field Science Research: 2018 update. MLTJ 2018; 8(3): $305-307$. 